\title{
Adaptive MAC protocol for wireless sensor networks in periodic data collection applications
}

\begin{abstract}
In this paper, we propose a new medium access control (MAC) protocol for wireless sensor networks for environmental monitoring applications. The proposed MAC scheme is specifically designed for wireless sensor networks which have periodic traffic with different sampling rates. In our protocol design, only sink can start and maintain synchronization and also determine the time schedule for all other nodes in the network. We discuss the design of TA-PDC-MAC protocol and provide a comparison with the previous PDC-MAC protocol through simulation. Under different traffic generation rate, our protocol outperforms the previous one in terms of energy consumption, packet loss rate and packet delay.
\end{abstract}

Keyword: Medium access control (MAC) protocol; Wireless sensor networks; TA-PDCMAC protocol; Protocol designs 